\title{
Collaborative capture of geometry rationale
}

\author{
M. Klein \\ Center for Coordination Science \\ MIT Sloan School of Management One Amherst Street E40-169 \\ Cambridge MA 02139 \\ Telephone: +1 (617) 253-6796; Fax: +1 (617) 253-4424 \\ E-mail: m_klein@mit.edu
}

\begin{abstract}
When an artefact is designed the typical output consists of documents describing the final result of a long series of deliberations and trade-offs by the participants of collaborative design (AKA concurrent engineering) teams. The underlying intent and logical support (i.e. the rationale) for the decisions captured therein is usually lost, or is represented at best as a scattered, difficult-to-access collection of paper documents and designers' recollections. This is particularly true for geometric, as opposed to preliminary, design rationale. Rationale information is critical, however, for important collaborative design functions such as team memory, design retrieval and negotiation. This paper describes an evolving web-based tool designed to greatly improve capture and access of preliminary and geometric design rationale in concurrent engineering teams, based on a marriage of rationale capture, world-wide web, $\mathrm{CAD}$ and feature-based design technology.
\end{abstract}

\section{Keywords}

CAD, concurrent engineering, geometric design, web, collaboration 


\section{THE CHALLENGE: CAPTURING RATIONALE FOR DESIGN GEOMETRY}

When an artefact is designed (in this paper, the word "design" is taken to denote all aspects of an artifact's definition, including the requirements, geometric definition, materials, manufacturing plan, supporting documentation and so on) nowadays the typical output of this activity includes blueprints, CAD files, manufacturing plans and other documents describing the final result of a long series of deliberations and trade-offs by the participants of concurrent engineering (CE) teams. The underlying intent and logical support (i.e. the rationale) for the decisions captured therein is usually lost, or is represented at best as a scattered collection of paper documents, project and personal notebook entries as well as the recollections of the artefact's designers. This information can be very difficult to access and is represented such that computers can provide little support for managing and utilising it. This design rationale information is critical, however, for supporting important collaborative design functions like structured design space exploration (Lee and Lai, 1991, MacLean, Young, Bellotti, and Moran, 1991, McCall, 1987), team memory (Balzer, 1984), conflict management (Klein and Lu, 1991, Klein, 1997), and design retrieval (Mostow and Barley, 1987).

This gap is particularly evident for the important area of design geometry. While technology has been developed for capturing rationale for non-geometric design decisions (Klein, 1993),or domain-specific geometric design decisions (Fischer, McCall, and Morch, 1989), no generic geometry rationale capture approach has previously been developed.

This paper describes results of an ongoing effort to greatly improve capture and access to both preliminary and geometric design rationale in distributed multifunctional collaborative design (also known as concurrent engineering) teams. The essence of our approach is to extend an existing design rationale representation to include user-defined geometry features, and to integrate CAD and World Wide Web technology to support the viewing and updating of this information by design team members. Our ideas are currently instantiated in a Web-based tool called CDeSS (the Collaborative Design Support System). The remainder of this paper will describe the system and consider its strengths, weaknesses, and opportunities for further development.

\section{THE C-DESS SYSTEM}

The goal of C-DeSS is to allow design team members to collaboratively access and update an evolving design as well as its underlying rationale. The system uses the following simple architecture (Figure 1): 


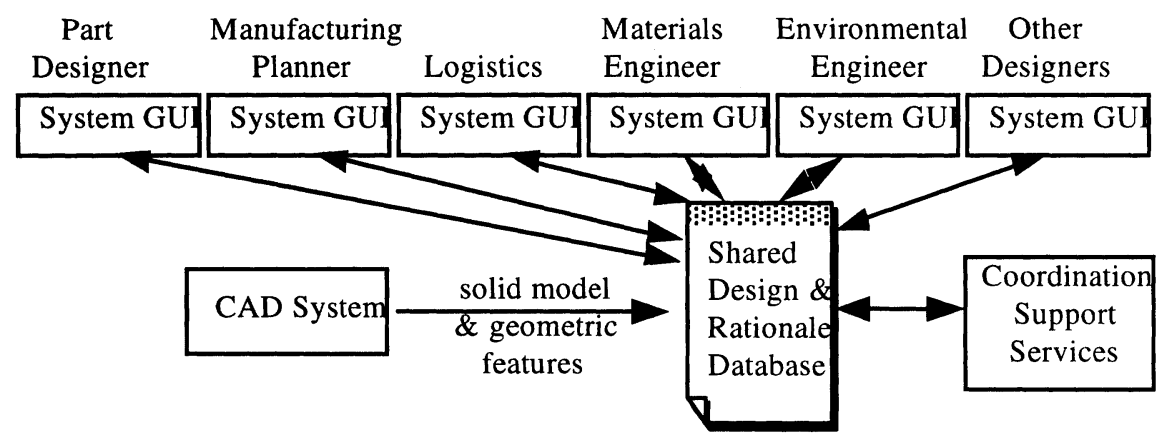

Figure 1: C-DeSS system architecture.

Design decisions and their rationale are stored in a shared database using a semiformal representation developed by the author over the last five years (Klein 1992, 1993). This data is accessed and updated by design team members using graphical user interfaces on standard Web browsers. Preliminary (i.e. non-geometric) design decisions, as well as all rationales, are entered directly via the browsers, while geometry data is defined via CAD systems (we used the Unigraphics CAD tool) and translated into 2D (GIF) and 3D (VRML) formats annotated with user-defined geometric features. These images, once entered into the shared design database, can be viewed over the Web and provide a visual "index" to which one can attach geometry rationale.

In the following sections we will consider the representation used to capture design rationale, as well as the user interface that allows design team members to access and update this information.

Design/Rationale Representation: The heart of C-DeSS is a semi-formal representation for capturing design decisions and their rationale. We will review the basics of this language as a necessary backdrop for discussing the novel contribution of this paper - the geometric rationale capture components.

The C-DeSS representation language captures designs using a vocabulary consisting of entities as well as claims about these entities. A pre-defined vocabulary of relational claims describes relationships between assertions. Any claim can serve as part of the rationale for another claim, so we can make claims about the design (e.g. module-1 has-submodule module-2), claims describing rationale for design decisions (e.g. value-1 is-derived-from procedure-1), claims concerning why we should believe a rationale or not (e.g. [A is-derived-from B] isdenied-by $\mathrm{C}$ ), and so on recursively. There is also an all-purpose text claim used to capture information not otherwise expressible. The net result of describing designs and rationale in this way is a graph of entity and text claim instances connected by relational claims. 
Non-geometric design decisions are captured using entities like modules, interfaces, attributes and values as well as relations such as has-submodule, hasattribute, has-interface and has-connection (Figure 2):

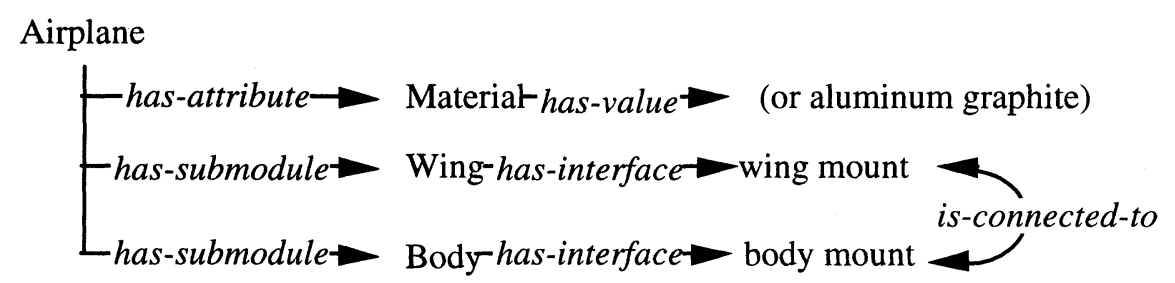

Figure 2: An example of preliminary design decisions.

Rationale is captured using a vocabulary that includes entities such as "procedure" and relations such as "supports", "denies", "has-result" (relating a claim to the procedure that generated it) and so on. To get more detail, including examples, of the preliminary design and rationale components of the language used in C-DeSS, please refer to (Klein, 1993) and (Klein 1992)

Let us now turn to the key contribution of this work; the language extensions developed to capture rationale for geometric decisions. C-DeSS captures geometric decisions as a set of claims about geometric features and their spatial interrelationships (see Figure 3; in this figure, entities are represented in plain font, while the relations that can hold between these entities are represented as directed arcs with italic labels:

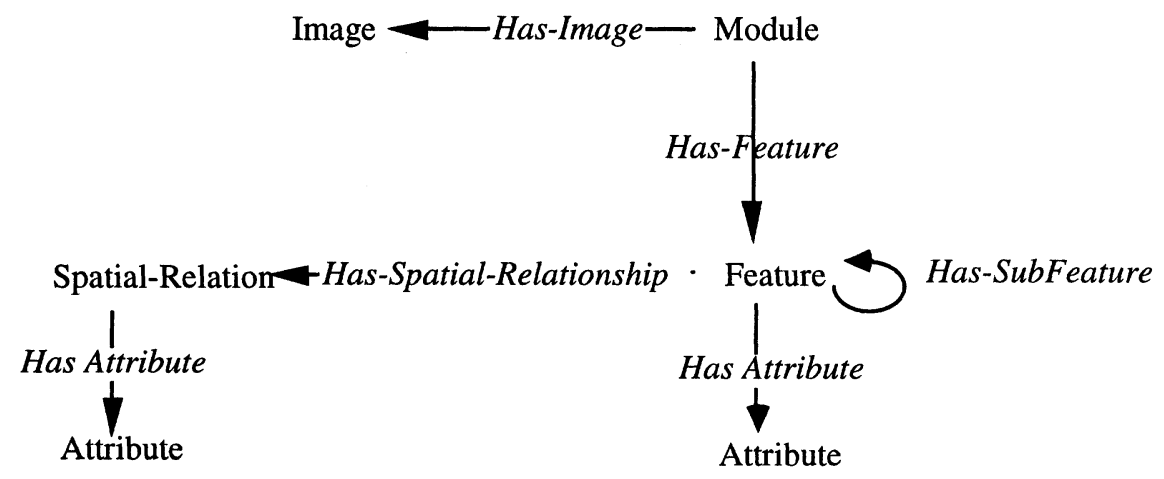

Figure 3: Geometry capture entities and relationships. 
A "geometric feature" is any meaningful geometry fragment such as a slot, hole, edge, shaft center line etc. (Salomons, v. Houten and Kals, Shah, 1991). Any module in a product design can have one or more features. Features can have attributes (such as the width of a slot, or the diameter of a hole) and can be composed of smaller "sub-features". Features can also be inter-related by "spatial relationships" that express meaningful geometric relationships like "is-parallel-to" or "is-separated-from". Spatial relations, like features, can have attributes; one can represent in this way, for example, the "distance" attribute for the "is-separatedfrom" relation. This representation is capable of fully capturing product geometry if necessary.

Since features and spatial relations are claims, they can have rationale attached to them just like any other kind of design claim. One can, for example, attach rationale to the "diameter" attribute of a hole feature, or to the "angle" attribute of an "is-skewed-from" spatial relation. One can even attach rationale to the claim that a part has a given feature or relation at all.

Features appear to be largely in the eye of the beholder, in the sense that features that are meaningful from one perspective, such as manufacturing, may differ than those that are useful when discussing geometry from, for example, a functional perspective. The C-DeSS language as a result does not mandate any particular feature taxonomy but rather allows users to define geometric features as they like, by selecting a geometric region (e.g. using a CAD package) and defining a corresponding feature (along with its name and attributes) as they like. A spatial relationship, similarly, is created by selecting the two or more features to be related, and describing the name and attributes for the new relation. The features so defined do not need to fully capture the geometry of the design being considered; their role is solely to provide anchors for attaching rationale to those aspects of the design geometry the design team members are interested in.

User Interface: The web-based user interface pictured below (Figure 4) allows design team members to access and annotate the rationale for a products' (preliminary or geometric) design. The interface consists of four panes: the current set of design alternatives on the upper left, a geometry display pane on the upper right, a textual details pane on the lower right, and a menu pane on the lower right. ...The system is used as follows. Once a designer has defined a candidate geometry using a standard CAD system, the CAD data is translated into one or more 2D (image-mapped GIF) or 3D (VRML) images with hot regions corresponding to the user-defined geometric features. Any design team member can then click on an image hot region to get more detailed information on the corresponding feature in the "details" pane (by selecting "show rationale" from the menu pane), or attach rationale to the feature or associated spatial relations (e.g. by selecting "add support" from the menu pane). 


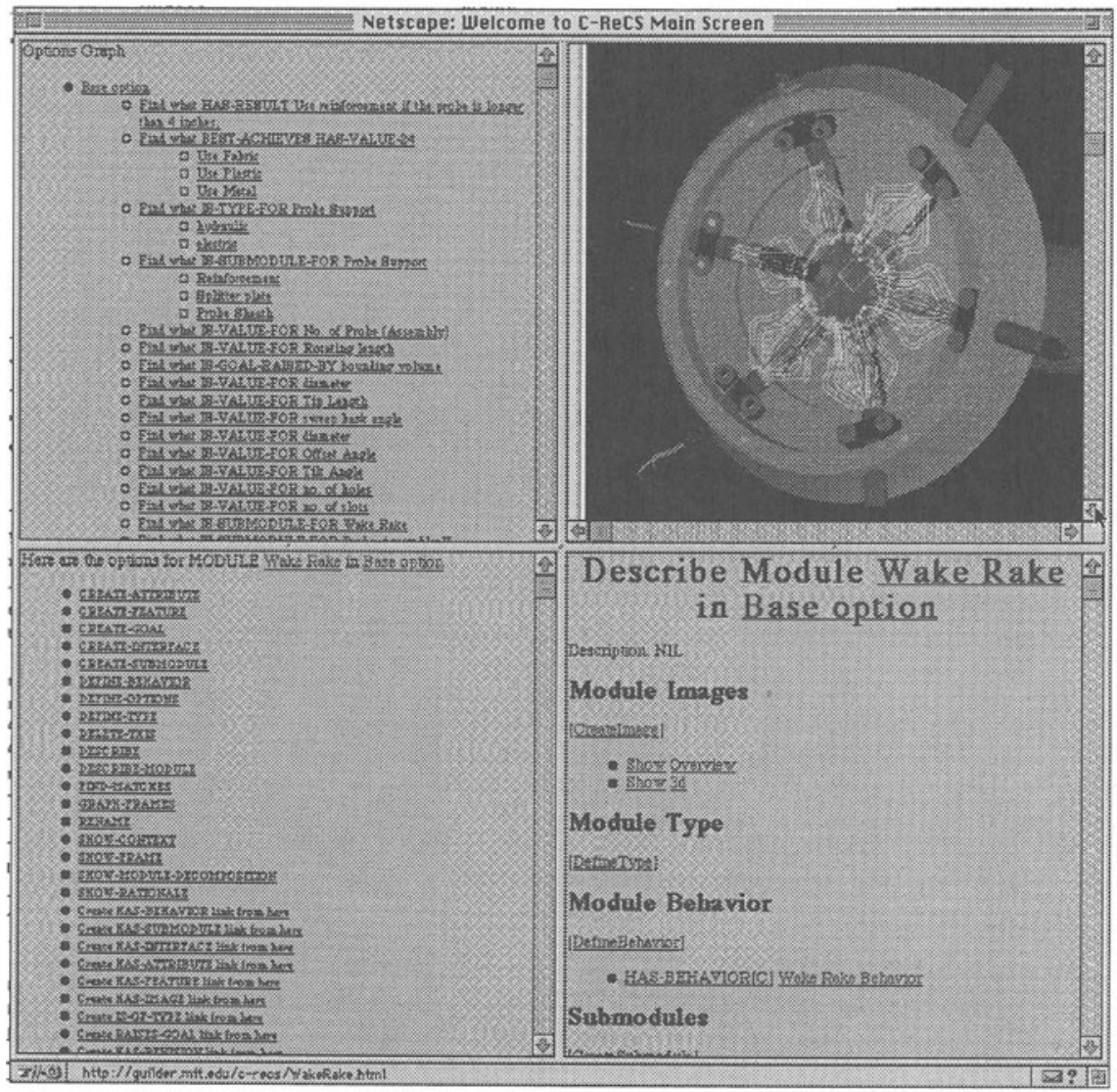

Figure 4: The C-DeSS user interface.

One can for example click on a probe in the image above to access the "probe column" feature, and from there learn or add to the rationale for the probe's length. Rationale added by any team member immediately becomes available for viewing and further annotation by any other team member. In this way, an entire design team can carry on a distributed and even asynchronous discussion about a design and its rationale in a way that leaves a record for future use.

\section{EVALUATION AND FUTURE GROWTH}

This system has been evaluated by using it to capture the design and associated rationale for a fluid-field measurement device developed at Pennsylvania State University's Applied Research Lab; this device is affectionately called the "Wake 
Rake" since it is typically used to assess torpedo and ship wakes. We interviewed the chief designer of the Wake Rake to develop an understanding of its design, and entered a representative collection of 2D and 3D images of the Wake Rake into the shared design database along with hot regions for what we judged to be the interesting features. Several engineers on our team then used C-DeSS over a period of several weeks to browse the design and annotate it with rationale based on the interviews we did as well as their individual engineering expertise. Their feedback drove an iterative process of user interface refinement and further evaluation.

Our experience suggests that our basic metaphor is a natural one for capturing and accessing geometric design rationale information. The engineers were readily able to frame design rationale in terms of geometric features, and appreciated being able to access the rationale for a part of the product geometry simply by clicking on it. It was found that both 2D and 3D displays of product geometry were useful, and the engineers typically appreciated having multiple views, highlighting different features, for any given part. It was also found that the engineers valued having hot regions that allow them to navigate between different views, for example by clicking on a wake rake probe to get a more detailed picture of the probe and its characteristic features.

The preliminary results are thus encouraging. We have shown that a novel integration of existing design rationale, feature-based geometry, Web and CAD technology has significant promise for addressing the important challenge of facilitating rationale capture in collaborative design settings, including teams that are distributed in time and space.

Much, however, remains to be done. A key weakness in the current implementation is the relatively poor integration between the CAD tool and the CDeSS system. Currently, one can only start defining features after the geometry has been fully defined in the CAD system and translated into GIF or VRML images. The hot regions corresponding to geometric features are then added by hand. It would clearly be better if features could be defined and annotated with rationale from within the CAD environment as the design geometry is being developed. Another problem is that since features are added post-hoc to the design geometry, there is no guarantee that they will remain important or even valid after even a minor change in the geometry. The only complete answer to this latter concern is to define the design in terms of features from the very start; most CAD systems, however, are not feature based. As a result, C-DeSS is currently best suited for discussing a few defined designs in detail, as opposed to quickly evaluating a number of evolving alternative geometries.

In addition to these technical weaknesses, the relatively informal evaluation we have done needs to be augmented by a more systematic one that includes a comparison between the C-DeSS approach, current practice in industry, and competing technological solutions such as standalone rationale capture tools like IBIS. This will likely lead to useful insights into the design of the C-DeSS representation and user interface. 


\section{ACKNOWLEDGEMENTS}

I would like to gratefully acknowledge the valuable contributions made by the other participants of this project, including Soonki Kweon, Paul Swanson, Mark Traband, Al Treaster and Mike Yukish, all at the Pennsylvania State University Applied Research Lab. This work was supported by the Applied Research Lab as well as by the Logistics Division of Armstrong Labs, Wright-Patterson AFB, Janet Peasant POC.

\section{REFERENCES}

J. Lee and K.-Y. Lai, (1991), "What's In Design Rationale?", Human-Computer Interaction, vol. 6, pp. 251-280.

A. MacLean, R. Young, V. Bellotti, and T. Moran, (1991), "Questions, Options and Criteria: Elements of a Design Rationale for User Interfaces", Journal of Human Computer Interaction: Special Issue on Design Rationale, vol. 6, pp. 201-250.

R. McCall, (1987), "PHIBIS: Procedurally Heirarchical Issue-Based Information Systems", Conference on Planning and Design in Architecture, Boston MA.

R. Balzer, (1984), "Capturing the Design Process in the Machine", Rutgers Workshop on Knowledge-Based Design Aids.

M. Klein and S. C.-Y. Lu, (1991), "Detecting and Resolving Conflicts Among Cooperating Human and Machine-Based Design Agents", The International Journal For Artificial Intelligence in Engineering.

M. Klein, (1997), "An Exception Handling Approach to Enhancing Consistency, Completeness and Correctness in Collaborative Requirements Capture", Concurrent Engineering Research and Applications.

J. Mostow and M. Barley, (1987), "Automated Reuse of Design Plans", International Conference on Engineering Design (ICED-87).

M. Klein, (1993), "Capturing Design Rationale in Concurrent Engineering Teams", IEEE Computer.

G. Fischer, R. McCall, and A. Morch, (1989), "Design Environments for Constructive and Argumentative Design", Computer-Human Interfaces (CHI89).

M. Klein, (1992), "DRCS: An Integrated System for Capture of Designs and Their Rationale", Second International Conference on Artificial Intelligence in Design (AID-92), Pittsburgh, Pennsylvania.

O. W. Salomons, F. J. A. M. v. Houten, and H. J. J. Kals, "Review of Research in Feature-Based Design", Journal of Manufacturing Systems, vol. 12, pp. 113132. 
J. J. Shah, (1991), “Assessment of Features Technology”, Computer Aided Design, vol. 23, pp. 331-343.

\section{BIOGRAPHY}

Dr. Mark Klein is a Research Associate in the MIT Center for Coordination Science, where he conducts research on co-ordination science including technology for conflict management, design rationale capture, process exception handling and workflow management. He has worked on advanced research and development in both industrial (Boeing, Hitachi) and academic (University of Illinois, Pennsylvania State University, MIT) settings. His professional activities include invited talks on three continents, chairing several international workshops, guest editing special journal issues on collaborative design as well as serving on numerous editorial boards and conference program committees. Mark has published over 40 papers. 